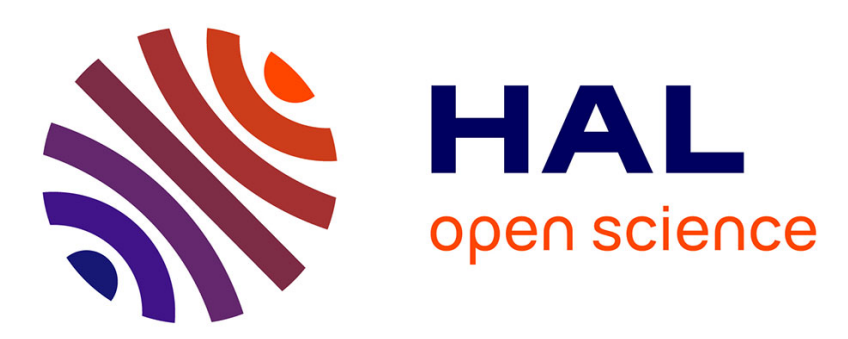

\title{
Concurrence entre établissements et choix de l'école : les personnels de direction mis à l'épreuve ? Gilles Combaz
}

\section{To cite this version:}

Gilles Combaz. Concurrence entre établissements et choix de l'école : les personnels de direction mis à l'épreuve ?. Education et Sociétés: Revue internationale de sociologie de l'éducation, 2013, 31 (1), pp.157-170. 10.3917/es.031.0157 . hal-03354769

\section{HAL Id: hal-03354769 \\ https://hal.univ-lyon2.fr/hal-03354769}

Submitted on 27 Sep 2021

HAL is a multi-disciplinary open access archive for the deposit and dissemination of scientific research documents, whether they are published or not. The documents may come from teaching and research institutions in France or abroad, or from public or private research centers.
L'archive ouverte pluridisciplinaire HAL, est destinée au dépôt et à la diffusion de documents scientifiques de niveau recherche, publiés ou non, émanant des établissements d'enseignement et de recherche français ou étrangers, des laboratoires publics ou privés. 


\title{
CONCURRENCE ENTRE ÉTABLISSEMENTS ET CHOIX DE L'ÉCOLE : LES PERSONNELS DE DIRECTION MIS À L'ÉPREUVE?
}

\author{
Gilles Combaz
}

De Boeck Supérieur | Education et sociétés

\author{
$2013 / 1-n^{\circ} 31$ \\ pages 157 à 170
}

\section{ISSN 1373-847X}

Article disponible en ligne à l'adresse:

http://www.cairn.info/revue-education-et-societes-2013-1-page-157.htm

Pour citer cet article :

Combaz Gilles, « Concurrence entre établissements et choix de l'école : les personnels de direction mis à l'épreuve? »,

Education et sociétés, 2013/1 n³1, p. 157-170. DOI : 10.3917/es.031.0157

Distribution électronique Cairn.info pour De Boeck Supérieur.

(c) De Boeck Supérieur. Tous droits réservés pour tous pays.

La reproduction ou représentation de cet article, notamment par photocopie, n'est autorisée que dans les limites des conditions générales d'utilisation du site ou, le cas échéant, des conditions générales de la licence souscrite par votre établissement. Toute autre reproduction ou représentation, en tout ou partie, sous quelque forme et de quelque manière que ce soit, est interdite sauf accord préalable et écrit de l'éditeur, en dehors des cas prévus par la législation en vigueur en France. II est précisé que son stockage dans une base de données est également interdit. 


\section{Concurrence entre établissements et choix de l'école : les personnels de direction mis à l'épreuve?}

\section{Gilles Combaz}

Éducation, cultures et politiques, Université Lumière Lyon 2 Institut des sciences et des pratiques d'éducation et de formation Campus des Berges du Rhône, 86 rue Pasteur, 69365 Lyon Cedex 07 gilles.combaz@univ-lyon2.fr

E n France, la question de la concurrence entre les établissements scolaires et celle du choix de l'école par les familles ont fait l'objet de nombreux travaux sociologiques au cours des cinq dernières années. Les choix politiques mis en œuvre au cours de cette période expliquent en partie l'intérêt que les chercheurs ont porté à ces thématiques. Après l'élection présidentielle de 2007, le ministre de l'Éducation nationale a proposé d'assouplir les règles d'affectation des élèves dans les établissements publics. Auparavant, à l'exception de ceux qui avaient choisi l'enseignement privé, les parents étaient contraints d'inscrire leurs enfants dans l'établissement public du secteur géographique dont ils dépendaient. Créé en 1963, ce dispositif -appelé carte scolaire- avait pour principaux objectifs de prévoir la gestion des personnels et des équipements et de limiter les disparités dues à la composition sociale des établissements (Meuret et al. 2009). Jugée inadaptée à la situation contemporaine, cette mesure a été progressivement assouplie dès le mois de juin 2007. Le site Internet du ministère de l'Éducation nationale précise alors que "cette nouvelle liberté offerte aux familles aboutira à améliorer la mixité sociale et géographique des établissements ainsi qu'à renforcer l'égalité des chances". Cette orientation a suscité le développement de nombreuses recherches sociologiques (Ben Ayed 2009, 2011, Merle 2010, 2011a, Oberti 2007, Poupeau\& François 2008, van Zanten 2009). La question du choix de l'école par les familles est liée à celle de la concurrence,la plus grande liberté accordée aux parents pouvant conduire à une spécialisation des établissements du point de vue de leur recrutement social. Ils se trouvent indirectement en rivalité par le biais du type d'élèves qu'ils sont susceptibles d'attirer (Broccolichi et al. 2010, Merle2011b). 
Aucune des études mentionnées n'aborde la façon dont les personnels de direction appréhendent les changements mis en œuvre. Pour la période couvrant les années 1990, deux recherches ont tenté d'analyser les tensions ressenties par les chefs d'établissement confrontés au développement de logiques de concurrence et au consumérisme éducatif. La première, centrée sur une trentaine d'établissements, permet de comparer la situation de la France et celle de la GrandeBretagne (Ball \& van Zanten 1998). La seconde prend appui sur une enquête nationale qui a permis d'interroger 654 responsables de collèges publics (Combaz 2003). Ces deux études sont désormais datées. Depuis le début des années 2000, le statut des personnels de direction et les missions qui leur sont confiées ont évolué (Pélage 2003). Le vivier de recrutement a également changé de composition : les enseignants restent encore majoritaires mais d'autres catégories de personnels accèdent à la fonction (conseillers principaux d'éducation, conseillers d'orientation). Le concours est ouvert à des fonctionnaires de catégorie A extérieurs à l'Éducation nationale. Avant de devenir chefs d'établissement, certains ont exercé une autre profession dans le secteur privé. L'hypothèse peut être faite que cette ouverture du recrutement a pu modifier l'éventail des valeurs et des conceptions sous-jacentes aux pratiques professionnelles des personnels de direction.

À l'époque où les procédures de carte scolaire étaient encore appliquées, des travaux avaient souligné les dérives de certains chefs d'établissement de lycées élitistes soucieux de recruter les meilleurs élèves et souhaitant s'assurer la bienveillance des familles socialement favorisées (Laforgue 2004). Avec l'assouplissement de la carte scolaire, il est plausible de supposer que ces attitudes ont pu se développer sans pour autant concerner l'ensemble des personnels de direction. Chercher à attirer les meilleurs élèves en vue d'améliorer les résultats et l'image de marque de l'établissement ne constitue pas nécessairement l'objectif ultime de tout personnel de direction du secteur public. Mais comme le suggère Merle, "les élèves d'un bon niveau scolaire, centrés sur un objectif de réussite, facilitent la gestion d'un établissement et rehaussent la réputation de celui-ci alors que, pour un chef d'établissement, la recherche de la mixité sociale, loin de lui offrir un bénéfice valorisé institutionnellement, est une source de difficulté potentielle" (Merle2011b, 161).

Dans cette situation, il importe d'étudier de quelle façon les chefs d'établissement se placent par rapport aux évolutions qui viennent d'être décrites et qui, a priori, peuvent générer des tensions nouvelles. Ceci paraît d'autant plus important que "l'administration de l'éducation est passée de la normalisation bureaucratique des actes à l'enrôlement de la subjectivité, à l'engagement, au renforcement du contrôle de la loyauté” (Lang 2005, 96). 


\section{Points d'appui théoriques}

ouvent considéré comme la cheville ouvrière, le chef d'établissement est sol$\checkmark$ licité par une multitude de partenaires ne poursuivant pas nécessairement les mêmes objectifs : familles, collectivités territoriales, autorités de tutelle, enseignants, etc. Eu égard aux évolutions décrites ci-dessus, le personnel de direction peut se trouver en situation de porte-à-faux et tiraillé entre plusieurs logiques relativement contradictoires : exigence de justice sociale, recherche d'efficacité, attention portée à la demande des familles, respect des directives ministérielles. Ces différentes logiques renvoient à des univers de référence qu'il convient de caractériser sociologiquement. Pour cela, nous avons pris appui sur les modèles que Derouet (1992) a élaborés à partir des travaux de Boltanski \& Thévenot (1987). Parmi les cinq proposés, trois sont retenus : le modèle de l'intérêt général, le modèle marchand et le modèle de l'efficacité. Ils ont été sélectionnés parce qu'ils correspondent bien aux principes de justice qui peuvent guider les conceptions relatives au choix de l'école et à la concurrence entre les établissements.

Le modèle de l'intérêt général correspond aux principes essentiels régissant le service public. Les aspirations individuelles passent au second plan au profit du bien commun. L'égalité de traitement de tous les usagers constitue un objectif prioritaire. Pour le système éducatif, ceci peut se traduire par des mesures destinées à limiter les inégalités sociales de scolarisation et les ségrégations par l'école. Formellement, les procédures de sectorisation correspondent en partie à cette orientation.

Le modèle marchand se situe quasiment à l'opposé du premier. Il ne s'agit pas ici de contribuer à l'élaboration de l'intérêt général mais de répondre à la demande des individus. Le processus de désectorisation amorcéen 2007 se situe dans cette logique : il faut que les familles puissent choisir librement l'établissement dans lequel elles souhaitent scolariser leurs enfants. Les établissements doivent être suffisamment attractifspour éviter les comportements de fuite ou d'évitement de certaines familles souhaitant offrir les meilleures conditions de scolarisation à leurs enfants. Comme signalé plus haut, cette logique met indirectement en concurrence les établissements.

Le modèle de l'efficacité rend compte des efforts entrepris pour rationaliser l'organisation scolaire et la rendre plus performante. Il permet de décrypter les transformations intervenues dans le cadre des nouveaux modes de régulation des systèmes éducatifs. Ainsi, l'obligation pour l'école de rendre des comptes, la montée en puissance de l'évaluation et le pilotage par les objectifs et les résultats représentent des orientations qui correspondent bien au modèle de l'efficacité. 
Sans qu'ils apparaissent explicitement comme tels, ces trois modèles sont susceptibles de constituer des référentiels sur lesquels les personnels de direction prennent appui pour développer leur réflexion.

Certains analystes critiques attirent l'attention sur les liens qu'on peut établir entre les transformations qui ont affecté le fonctionnement du système éducatif et la montée en puissance du modèle marchand et du modèle de l'efficacité. Pour ces auteurs, ce phénomène marque le développement d'un courant néolibéral en éducation (cf. notamment Laval 2004). Il convient cependant d'être prudent car la notion de libéralisme est fréquemment employée de façon imprécise, laissant planer le doute sur la référence aux principes qui fondent l'économie libérale : le marché, l'initiative privée et la libre concurrence. En toute rigueur, le fonctionnement du système éducatif français ne repose pas sur de tels principes. Aussi, lorsque le terme de "conceptions libérales" est utilisé ici, c'est pour sa portée métaphorique. Ceci paraît particulièrement pertinent lorsqu'il s'agit d'évoquer la plus grande liberté accordée aux familles pour le choix de l'école ou pour faire référence aux logiques de concurrence entre les établissements.

\section{Méthodologie}

$\mathrm{N}$ otre recherche prend appui sur un double dispositif méthodologique : une enquête nationale par questionnaire et une série d'entretiens. À partir d'un échantillonnage aléatoire, 2400 établissements du second degré (secteur public) ont été tirés au sort (800 collèges, 250 lycées d'enseignement général et technologique et 150 lycées professionnels). Pour chaque établissement, deux questionnaires ont été envoyés : un pour le chef et un pour l'adjoint. Après deux relances, nous avons reçu 332 questionnaires exploitables (soit un taux de retour de 13,8\%). La partie du questionnaire utilisée pour la présente recherche est consultable en annexe. Les comparaisons établies avec des données nationales exhaustives (Ministère de l'Éducation nationale 2010) montrent que la représentativité de notre échantillon est satisfaisante eu égard aux caractéristiques habituellement prises en considération : le sexe, l'âge, les qualifications académiques et la répartition selon le type d'établissement. Pour compléter le volet quantitatif, vingt entretiens ont été menés dans trois académies : Paris, Versailles et Poitiers.

Une partie de notre questionnement portait sur les deux thématiques abordées dans le cadre de cet article : l'assouplissement de la carte scolaire et la concurrence entre les établissements. Nous avons invité les personnels de direction à réagir par rapport à neuf propositions qui renvoient à un éventail d'opinions susceptibles de correspondre aux intérêts et aux conceptions défendus par chaque catégorie d'acteurs concernés : autorités de tutelle, chefs d'établissement, 
responsables politiques, familles, enseignants, etc. Ces propositions illustrent les univers de référence décrits plus haut. Pour chacune d'entre elles, quatre modalités de réponse ont été présentées : de "Tout à fait d'accord" jusqu'à "Pas du tout d'accord" (voir la liste des propositions en annexe). Certains personnels de direction n'ont pas répondu à cette série de questions $(\mathrm{N}=9)$. Dans le questionnaire, les personnes interrogées pouvaient compléter leurs réponses par un commentaire de quelques lignes. En outre, l'entretien offrait la possibilité aux chefs d'établissement de développer plus longuement leurs positions.

Les données quantitatives ont fait l'objet d'un traitement statistique multidimensionnel, prenant en compte simultanément l'ensemble des réponses fournies (neuf propositions, 36 modalités). En suivant la démarche proposée par Lebart, Morineau\& Piron (2000), les méthodes factorielles et les méthodes de classification ont été utilisées conjointement. Ceci a facilité l'élaboration d'une typologie permettant de regrouper les personnes enquêtées en classes homogènes du point de vue de la nature des réponses données. La procédure choisie dans le logiciel utilisé (SPAD) regroupe les individus de la population en suivant un algorithme de classification fondé sur le critère d'agrégation de la variance (critère de Ward). Les individus sont rassemblés dans le même groupe de façon à minimiser la variance interne de chaque classe et à maximiser la variance entre les classes. La première étape a consisté à réaliser une analyse factorielle de correspondances multiples sur les 36 modalités de réponse. Au cours de la seconde étape, une classification ascendante hiérarchique a été mise en œuvre sur les facteurs en vue d'élaborer une typologie. Un certain nombre de variables indépendantes ont été prises en considération : le sexe, l'âge, l'ancienneté dans la fonction, le statut (chef ou adjoint), le type de formation initiale.

Les résultats issus de ce traitement statistique sont illustrés par les commentaires proposés et des extraits d'entretiens. Ceci permet d'identifier les arguments qu'utilisent les chefs d'établissement pour justifier leurs réponses.

\title{
Résultats
}

\begin{abstract}
$\Delta$
l'issue du traitement des données quantitatives, trois regroupements ont été envisagés : 3, 4 et 9 classes. Le troisième regroupement a été préféré aux deux premiers dans la mesure où il permet de faire émerger des classes plus homogènes et des nuances plus nombreuses. L'analyse des données révèle trois grands profils. Un quatrième profil apparaît mais il s'agit d'un cas un peu à part, comme nous le verrons par la suite. Aucune des variables indépendantes prises en considération n'est associée de manière significative à une ou plusieurs classes de la typologie.
\end{abstract}




\section{Un fort attachement aux principes du service public}

Trois classes (classes 7, 8 et 9. $\mathrm{N}=160$, soit 49,5\% de l'ensemble. Cf. tableau en annexe)sont regroupées sous cette appellation. À signaler que la classe 7 se distingue des deux autres par des opinions moins tranchées (emploi plus fréquent des modalités "Plutôt d'accord" ou "Plutôt pas d'accord").

Les personnels de direction regroupés dans ces trois classes se démarquent nettement des autres par le souci de défendre les principes du service public et le fait de prendre de la distance vis-à-vis de conceptions jugées sans doute trop libérales. Ils dénoncent fréquemment les dérives inhérentes à la montée du consumérisme éducatif. Ainsi, à propos de la plus grande liberté accordée aux parents pour choisir l'établissement dans lequel elles souhaitent scolariser leurs enfants, l'un d'eux mentionne: "Dans les faits, cela débouche sur des pratiques consuméristes de la part des familles qui choisissent leur établissement comme on choisit une voiture. En lieu et place de la consommation de carburant ou des options du véhicule, on trouve les résultats aux examens et la liste des voyages scolaires" (Homme, 40 ans, principal adjoint de collège). D'autres soulignent le risque de mettre en péril la mixité sociale. L'assouplissement de la carte scolaire pourrait alimenter le processus de "séparatisme social" mis au jour par les travaux de Maurin (2004) : "Cela entérine de manière peu républicaine et démagogique la tentative de repli sur soi des classes moyennes et supérieures, quand l'école est un lieu de passage obligé permettant précisément à tous de s'y mêler et d'y vivre ensemble" (Homme, 59 ans, proviseur adjoint de lycée). Comme le signale Ben Ayed (2009), le principe de mixité sociale associé à la carte scolaire renvoie à un enjeu de socialisation. Certains chefs d'établissement le signalent en utilisant des formules qui peuvent paraître excessives. Ainsi, en évoquant le processus de désectorisation, l'un d'eux écrit : "Il y a de l'apartheid dans cette idée-là. L'homme ne se définit pas seulement par sa réussite scolaire. Son intégration dans un groupe hétérogène est plus importante" (Homme, 60 ans, proviseur de lycée). Dans les grandes agglomérations, l'imbrication des ségrégations urbaines et des ségrégations scolaires mise en évidence par les recherches d'Oberti (2007) est également mentionnée : "Il serait judicieux d'avoir une politique de la ville mettant fin à la ghettoïsation de certains quartiers" (Femme, 40 ans, principale adjointe de collège). À cet égard, plusieurs personnes interrogées évoquent les difficultés rencontrées par les établissements qui ont été construits au sein de cités dites sensibles. Dans ce cas, l'objectif de mixité sociale paraît singulièrement compromis.

Les personnels de direction des classes 8 et 9 se révèlent relativement intransigeants vis-à-vis des alternatives envisageables, notamment la possibilité offerte aux familles de choisir parmi trois ou quatre établissements situés dans une aire géographique soigneusement délimitée. Ils acceptent cependant de prendre en considération certains motifs de dérogation jugés importants : "Il faut revenir à 
une application stricte de la carte scolaire en examinant les motifs de dérogation recevables pour y répondre favorablement" (Femme, 57 ans, principale de collège).

L'idée que l'abandon de la carte scolaire pourrait représenter un élément stimulant dans la mesure où l'établissement doit rester actif pour éviter les comportements de fuite, suscite une certaine méfiance, voire de l'hostilité. La réputation d'un établissement ne se construit pas nécessairement à partir d'éléments objectifs permettant d'apprécier sa réelle "valeur ajoutée" en termes d'acquisitions scolaires. Les mises en garde à ce sujet sont parfois formulées de manière directe : "Il faut faire très attention parce que les parents mesurent souvent la valeur d'un établissement au nombre de voyages à l'étranger, aux sorties, aux actions 'blingbling' plus qu'en termes de pédagogie et de stratégies d'apprentissage mises en place" (Femme, 52 ans, principale de collège).

Les personnels de direction des classes 7, 8 et 9 se montrent également opposés à l'idée que la concurrence entre les établissements permettrait d'accroître leur "efficacité" et leur "rendement". À ce propos, l'un d'entre eux écrit : "Un établissement scolaire n'est pas une entreprise à but lucratif. Il ne doit pas être jugé en termes de 'rendement' mais plutôt encouragé et aidé au lieu d'être mis en concurrence” (Femme, 41 ans, principale adjointe de collège). Un autre chef d'établissement souligne les effets potentiellement contre-productifs de la concurrence : "Cela rendrait sans doute les personnels de direction moins coopérants. Ce qui nuirait aux réflexions collectives et à la mise en place de dispositions communes comme cela se fait dans les bassins" (Femme, 45 ans, principale de collège). La proposition selon laquelle la concurrence entre établissements pourrait être envisagée à condition qu'elle soit strictement encadrée par les autorités de tutelle ne fait pas non plus l'unanimité, loin s'en faut. Manifestement, les administrations déconcentrées ne semblent pas inspirer une confiance absolue, comme en témoigne l'une des personnes interrogées : "Comment les autorités de tutelle pourraient-elles contrôler cela ? C'est une utopie. Elles n'ont qu'une connaissance de surface de ce qui se passe réellement dans les établissements" (Femme, 52 ans, principale de collège).

Les opinions des personnels de direction des classes 7, 8 et 9 divergent quelque peu au sujet de la dernière proposition (P9) qui suggère de remplacer le terme de concurrence par la notion d'émulation. Une partie des chefs d'établissement de la classe 9 dénonce une forme d'hypocrisie en indiquant qu'il est dangereux de jouer sur les mots. Pour ces personnes, le terme d'émulation est encore trop connoté. Il laisse sous-entendre que les établissements sont en compétition les uns avec les autres.

\section{Des conceptions plutôt libérales}

Sous cette appellation sont regroupés les personnels de direction des classes 4, 5 et $6(\mathrm{~N}=68$ soit 21,1\% de l'ensemble). Comme dans le cas précédent, il y a, 
entre ces trois classes, des nuances d'appréciation qui paraissent importantes à conserver. À cet égard, les personnes de la classe 4 se montrent plus mesurées en soutenant des positions moins radicales que celles que défendent les chefs d'établissement des classes 5 et 6 .

Les personnels de direction de ces trois classes se révèlent très attachés à la liberté des familles pour le choix de l'établissement. Et certains d'entre eux n'hésitent pas à dénoncer l'hypocrisie qui consiste à penser que la sectorisation permet de faire respecter un des principes essentiels du service : l'égalité de traitement de tous les usagers. Lorsque la sectorisation était appliquée, seules les familles les plus “informées” réussissaient à la contourner. À présent, les règles sont plus claires : "L'égalité n'existait pas vraiment avec la carte scolaire. Désormais, l'évitement de certains établissements est plus transparent" (Femme, 55 ans, principale de collège). Plusieurs chefs d'établissement estiment que la liberté accordée aux familles demeure encore trop circonscrite : "La désectorisation a été avant tout une annonce médiatique visant à rassurer les parents. En fait, les critères sont sélectifs et, à l'usage, on constate que l'on est très loin de faire ce que l'on veut quand on est une famille" (Femme, 51 ans, proviseur de lycée).

Les personnels de direction des classes 4, 5 et 6 sont nombreux à penser que l'assouplissement de la carte scolaire peut être un élément stimulant dans la mesure où l'établissement doit rester "actif" pour conserver ses élèves. L'un d'eux indique: "Je pense que cette désectorisation va permettre d'avoir une meilleure lisibilité du fonctionnement des établissements qui vont obligatoirement se poser des questions. Celui qui va perdre des classes, des heures ou des financements va se retrouver confronté à un véritable challenge" (Homme, 54 ans, principal de collège). Certains semblent avoir totalement intégré cette logique et tentent d'anticiper pour limiter la perte d'élèves : "Dans les collèges, la question de l'évitement est cruciale. La direction doit fournir un gros travail de relation avec l'école primaire pour faire valoir les atouts de l'établissement" (Femme, 44 ans, principale de collège). L'assouplissement de la carte peut aussi représenter un moyen indirect pour stimuler l'investissement des professeurs : "Avoir des élèves 'captifs' [considérés comme tels avec la sectorisation] n'incitepas les enseignants à se remettre en cause. Cela ne les encourage pas à être créatifs et à être investis pour tous les élèves" (Homme, 54 ans, principal adjoint de collège).

Les personnels de direction des classes 4,5 et 6 adhèrent à l'idée que la concurrence entre les établissements peut contribuer à une meilleure "efficacité" et à un "rendement" plus élevé. Ils se montrent cependant prudents en soulignant les risques associés à une concurrence sans merci : "La concurrence est facteur de dynamisme mais elle doit être loyale" (Homme, 59 ans, principal). Pour les chefs d'établissement de la classe 4, elle peut exister à condition qu'elle soit encadrée par les autorités de tutelle. 
Les classes 5 et 6 se singularisent de deux manières. Pour la première, il s'agit d'un plein accord avec l'idée qu'il faut donner le choix aux familles entre trois ou quatre établissements sur une zone géographique bien délimitée. Pour la classe 6, il s'agit de substituer le terme concurrence à celui d'émulation qui paraît moins connoté et plus constructif.

La classe 6 représente un cas intermédiaire. Elle se définit essentiellement de deux manières : accorder plus de liberté aux familles pour le choix de l'établissement (en limitant cependant celui-ci : proposition P3. Cf. tableau en annexe); le souci que la concurrence entre les établissements soit contrôlée par les autorités de tutelle.

\section{Les garanties du service public assorties de quelques souplesses}

Au nombre de 67 (soit 20,7\% de l'ensemble), les personnels de direction regroupés sous cette appellation se caractérisent par des réponses qui se veulent nuancées (emploi quasi systématique des modalités "Plutôt d'accord" ou "Plutôt pas d'accord", cf. classes 1 et 2, tableau en annexe). Les chefs d'établissement appartenant à ces deux classes se montrent partagés au sujet de la liberté des familles pour choisir l'établissement. Selon qu'on se situe en tant qu'agent de l'institution ou en tant que parent, les positions fluctuent : "Je suis vraiment très partagée parce que si je réfléchis en tant que parent, je n'aurais peut-être pas envie d'envoyer mes enfants dans un collège en éducation prioritaire. Si je me positionne en tant que mère, j'aimerais bien avoir le choix. En même temps, si l'on permet ça, on enlève toute la petite mixité qui existait" (Femme, 44 ans, principale adjointe de collège). On fait également remarquer que toutes les familles ne possèdent pas nécessairement les compétences pour opérer un choix "éclairé". Certaines d'entre elles n'osent pas engager la démarche : "Attention, certaines familles de milieux modestes n'osent pas profiter de l'occasion qui leur est offerte" (Femme, 34 ans, principale adjointe de collège). Des travaux récents montrent effectivement que les familles socialement favorisées ont davantage bénéficié de l'assouplissement de la carte scolaire que les parents boursiers (Merle 2011a).

Les avis sont également partagés quant à la perspective d'offrir le choix entre trois ou quatre établissements situés dans une aire géographique délimitée. Les personnels de direction font remarquer que cette solution est essentiellement valable pour les zones urbaines. Ils attirent l'attention sur "Les difficultés rencontrées dans les régions rurales où le choix se fait avant tout en fonction des moyens de transport disponibles" (Homme, 37 ans, principal adjoint de collège).

Les chefs d'établissement des classes 1 et 2 ne se montrent pas radicalement opposés à l'idée que l'assouplissement de la carte scolaire puisse dynamiser l'établissement mais, là aussi, les opinions sont nuancées. Les disparités des moyens alloués paraissent constituer un obstacle : "À chacun d'être attractif avec un projet 
d'établissement stimulant, un contrat d'objectifs, une bonne communication. Mais cela semble quand même compliqué dans la mesure où d'un département à l'autre les moyens ne sont absolument pas les mêmes" (Femme, 37 ans, principale adjointe de collège).

La concurrence entre les établissements n'est pas non plus rejetée en bloc mais là encore, la prudence semble s'imposer : "Cela doit être conduit avec modération. Il y a certes des établissements qui s'endorment parfois sur leurs lauriers mais il y a aussi des établissements qui sont victimes de ceux qui se débarrassent de leurs difficultés" (Homme, 59 ans, principal de collège). La concurrence pourrait être acceptée à condition qu'elle soit contrôlée par les autorités de tutelle et qu'elle puisse s'établir sur des critères objectifs permettant d'apprécier réellement les performances de l'établissement (la notion de performance étant entendue dans un sens très large) : "Je pense qu'il faut que ce soit contrôlé par les autorités de tutelle et il faut qu'on arrive à une analyse de la performance des établissements. Et performant ne veut pas forcément dire avoir des super-taux de réussite aux examens. Est-ce que l'établissement est performant dans la prise en compte de la diversité des élèves ? Est-ce qu'il est performant dans le parcours qu'il offre aux élèves ?" (Femme, 52 ans, proviseur de lycée).

Les personnels de direction de la classe 1 se montrent particulièrement partagés au sujet de la proposition P6 ("La concurrence serait une excellente solution qui permettrait d'accroître "l'efficacité" et le "rendement" des établissements scolaires") : 33,3\% sont plutôt d'accord et $60 \%$ ne sont plutôt pas d'accord. Comme leurs collègues de la classe 2 , ils paraissent préférer le terme d'émulation à celui de concurrence. Le fait de travailler en complémentarité paraît beaucoup plus constructif qu'une concurrence stérile. Ceci semble particulièrement appréciable pour les établissements de petite taille : "Le travail en réseaux permet aux petits établissements d'offrir une vraie émulation, de rompre avec l'isolement et d'élargir l'offre de formation pour les familles" (Femme, 43 ans, principale de collège).

Regroupant 28 chefs d'établissement (soit 8,7\% de l'ensemble), la classe 3 représente un cas un peu à part des trois grands profils décrits jusqu'ici. Deux traits essentiels la caractérisent : le souci de préserver une certaine liberté pour le choix de l'école et l'idée que la concurrence entre établissements n'est pas nécessairement à bannir à condition qu'elle soit encadrée par les autorités de tutelle.

\section{Conclusion}

Les réactions dont nous venons de faire état montrent que les personnels de direction ne sont pas insensibles aux évolutions récentes qui ont affecté le choix de l'école par les familles et la concurrence entre les établissements. Une bonne partie d'entre eux se montresoucieuse des dérives pouvant être liées aux changements qui sont intervenus. Ils craignent que les principes essentiels du 
service public ne soient bafoués. Une minorité (20\%) se distingue par des conceptions plutôt libérales. Pour ces chefs d'établissement, l'assouplissement de la carte scolaire présente l'avantage de stimuler les établissements dans la mesure où ils doivent se montrer suffisamment attractifs pour conserver leurs élèves. Dans cette perspective, une certaine dose de concurrence paraît utile pour lutter contre l'inertie du système.

Quelles que soient les conceptions mises au jour ici, il semble que la loyauté des fonctionnaires interrogés soit mise à l'épreuve. Selon les environnements d'exercice, certains chefs d'établissement peuvent être enclins à jouer alternativement avec les règles informelles du marché scolaire et les principes du service public (van Zanten, 2009). Une recherche récente suggère que ce fonctionnement est entretenu par une forme de laisser faire émanant des autorités de tutelle (Broccolochi et al. 2010). Une étude antérieure réalisée sur la ville de Bordeaux avait déjà mis en évidence ce type de phénomène (Félouzis et al. 2005). Elle a montré que par rapport à la carte scolaire, les règles appliquées par l'inspection académique étaient relativement fluctuantes et les décisions prises relevaient souvent d'une gestion “à l'affectif”. Ceci a tendance à délégitimer les actions mises en œuvre. Comme l'ont montré certaines recherches (Lang 2005, van Zanten 2005), les administrations déconcentrées n'assument pas toujours les tâches de régulation intermédiaire qui leur incombent. Dans ces conditions, les personnels de direction risquent d'être en difficulté pour construire les compromis nécessaires à l'élaboration de l'intérêt général de l'établissement.

\section{Références bibliographiques}

BALL S. \& van ZANTEN A. 1998 "Logiques de marché et éthiques contextualisées dans les systèmes scolaires français et britanniques”, Éducation et Sociétés-1, 47-71

BEN AYED C. 2009 Le nouvel ordre éducatif. Mixité, disparités, luttes locales. Paris, PUF

BEN AYED C. 2011 "À qui profite le choix de l'école ?Changements d'établissement et destins scolaires des élèves de milieux populaires". Revue française de pédagogie-175, 39-57

BOLTANSKI L. \& THÉVENOT L. 1987 Les économies de la grandeur. Paris, PUF

BROCCOLICHI S., BEN AYED C. \&TRANCART D. 2010 École : les pièges de la concurrence. Paris, La Découverte

COMBAZ G. 2003 Les chefs d'établissement face aux paradoxes de l'école démocratique de masse. L'exemple des principaux de collèges publics en France. Revue des sciences de l'éducation-3, 629-648

DEROUET J.-L. 1992 École et justice. De l'égalité des chances aux compromis locaux? Paris, Métailié

FELOUZIS G., LIOT F. \&PERROTON, J. 2005 L'apartheid scolaire. Enquête sur la ségrégation ethnique dans les collèges. Paris, Seuil. 
LAFORGUE D. 2004 “Ce que la ségrégation scolaire doit à l'administration de l'Éducation nationale”. Revue française de pédagogie-148, 135-143

LANG V. 2005 La ligne hiérarchique de l'administration nationale à l'épreuve de la déconcentration. In Dutercq Y. coord. Les régulations des politiques d'éducation. Rennes, PUR, $85-97$

LAVAL C. 2004 L'école n'est pas une entreprise. Paris, La Découverte

LEBART L., MORINEAU A. \& PIRON M. 2000 Statistique exploratoire multidimensionnelle, Paris, Dunod

MAURIN E. 2004 Le ghetto français. Enquête sur le séparatisme social. Paris, Le Seuil

MERLE P. 2010 Structure et dynamique de la ségrégation sociale dans les collèges parisiens. Revue française de pédagogie-170, 73-85

MERLE P. 2011a La carte scolaire et son assouplissement. Politique de mixité sociale ou ghettoïsation des établissements ? Sociologie-1, 37-50

MERLE P. 2011b Concurrence et spécialisation des établissements. Une modélisation de la transformation du recrutement des secteurs d'enseignement public et privé. Revue française de sociologie-1, 133-169

MEURET D., BROCCOLICHI S. \&DURU-BELLAT M. 2009 La carte scolaire en France : un va-et-vient entre sectorisation et assouplissement. Problèmes politiques et sociaux-956, 13-16

MINISTÈRE DE LÉDUCATION NATIONALE 2010 Bilan social, Paris, France

OBERTIM. 2007 L'école dans la ville. Ségrégation, mixité, carte scolaire. Paris, Presses de la FNSP PÉLAGE A. 2003 La redéfinition du métier de chef d'établissement secondaire : changement statutaire, construction de l'engagement professionnel et épreuves pratiques. Revue française de pédagogie-145, 21-36

POUPEAU F. \& FRANÇOIS J.-C. 2008 Le sens du placement. Ségrégation résidentielle et ségrégation scolaire. Paris, Raisons d'agir

van ZANTEN A. 2005 La régulation par le bas du système éducatif : légitimité des acteurs et construction d'un nouvel ordre local. In Dutercq Y. coord. Les régulations des politiques d'éducation. Rennes, PUR, 99-117

van ZANTEN A. 2009 Choisir son école. Stratégies familiales et médiations locales. Paris, PUF

\section{Annexes}

\section{La carte scolaire}

En juin 2007, le ministre de l'Éducation nationale de l'époque, Monsieur Xavier Darcos, a proposé que les mesures de carte scolaire soient progressivement abandonnées.

Pour chacune des propositions suivantes (de P1 à P5), vous indiquerez si vous êtes tout à fait d'accord, plutôt d'accord, plutôt pas d'accord ou pas du tout d'accord.

Tout à fait d'accord P1++ Plutôt d'accord P1 +

Plutôt pas d'accord P1- $\quad$ Pas du tout d'accord P1- - 
P1 Ce processus de "désectorisation" est une bonne chose parce que cela permet aux familles de choisir plus librement l'établissement dans lequel elles souhaitent scolariser leurs enfants.

P2 Cette évolution présente des risques majeurs. Le système antérieur de la carte scolaire permettait de faire respecter l'un des principes essentiels du service public : l'égalité de traitement de tous les usagers.

P3 Une formule intermédiaire entre les deux alternatives ("désectorisation" ou "sectorisation") aurait sans doute été préférable (en laissant par exemple aux familles le choix entre trois ou quatre établissements sur une aire géographique soigneusement délimitée).

P4 L'abandon des mesures de carte scolaire va contribuer à la constitution d'un système éducatif à plusieurs vitesses. Certains établissements jugés attractifs par les parents vont pouvoir recruter de très bons élèves pendant que d'autres, jugés moins attractifs, vont scolariser des élèves cumulant plusieurs types de difficultés (scolaires, socio-économiques, etc.).

P5 La proposition 4 (ci-dessus) n'est valable que pour des cas particuliers d'établissements (très favorisés ou très défavorisés). Pour les autres cas (les plus nombreux), l'abandon de la carte scolaire représente un élément stimulant dans la mesure où l'établissement doit rester suffisamment actif pour être en mesure de conserver ses élèves.

\section{La question de la concurrence entre les établissements}

Dans le souci d'améliorer le fonctionnement du système éducatif français, on avance parfois l'idée qu'une "concurrence" plus forte entre les établissements scolaires serait nécessaire.

Pour chacune des propositions suivantes (de 1 à 4), vous indiquerez si vous êtes tout à fait d'accord, plutôt d'accord, plutôt pas d'accord ou pas du tout d'accord.
Tout à fait d'accord $\mathbf{P 6}++$
Plutôt d'accord P6 +
Plutôt pas d'accord P6-
Pas du tout d'accord P6- -

P6 Ce serait une excellente solution qui permettrait d'accroître l'“efficacité" et le "rendement"des établissements scolaires.

P7 Cette idée présente de nombreux inconvénients. En particulier, il apparaît que le développement de la "concurrence" entre les établissements scolaires pourrait conduire à la constitution d'une école à plusieurs vitesses (pôles d'excellence d'un côté et, de l'autre, établissements "ghettos” qui cumulent les handicaps).

P8 L'idée de développer la "concurrence" entre les établissements scolaires n'est pas forcément à rejeter à condition qu'elle soit strictement contrôlée par les autorités de tutelle pour éviter toute dérive.

P9 Le terme de concurrence n'est pas approprié pour caractériser les relations qui se nouent entre les établissements scolaires. Le terme d'émulation convient mieux. Dans cette perspective, les établissements peuvent fonctionner de manière complémentaire les uns par rapport aux autres sur une zone géographique précise. 


\begin{tabular}{|c|c|c|c|c|c|c|c|c|c|c|}
\hline \multicolumn{11}{|c|}{ Typologie des chefs d'établissement } \\
\hline & & & & & Classes & & & & & \\
\hline & $\mathbf{N}^{\circ} \mathbf{I}$ & $\mathbf{N}^{\circ} 2$ & $N^{\circ} 3$ & $N^{\circ} 4$ & $N^{\circ} 5$ & $N^{\circ} 6$ & $N^{\circ} 7$ & $N^{\circ} 8$ & $N^{\circ} 9$ & Ens. \\
\hline & $n=45$ & $n=22$ & $\mathrm{n}=28$ & $n=37$ & $n=14$ & $n=17$ & $n=42$ & $n=67$ & $n=51$ & $n=323$ \\
\hline \multicolumn{11}{|c|}{ Propositions relatives à la carte scolaire } \\
\hline $\mathrm{PI}++$ & $0,0,0$ & 9,1 & 0,0 & 0,0 & $\mathbf{5 7 , I * *}$ & $58,8 * *$ & 0,0 & 1,6 & 2,0 & 6,9 \\
\hline $\mathrm{PI}+$ & 24,4 & 13,6 & $51,9 * *$ & $78,4^{*}$ & 7,2 & 29,4 & $2,5 * *$ & $6,3 * *$ & $2,0 * *$ & 21,8 \\
\hline $\mathrm{PI}-$ & $60,0 * * *$ & 50,0 & 37,0 & $10,8 * *$ & 35,7 & $0,0^{* *}$ & $65,0 * *$ & 39,1 & $3,9 * *$ & 34,7 \\
\hline $\mathrm{PI}--$ & 15,6 *** & 27,3 & $11, I^{* *}$ & $10,8 * *$ & 0,0 & $11,8 * *$ & 32,5 & $53, I * *$ & $92,1 * *$ & 36,6 \\
\hline \\
\hline $\mathrm{P} 2++$ & 22,2 & 50,0 & 44,4 & $2,7^{* *}$ & 28,6 & $0,0^{* *}$ & $4,9 * *$ & $57,1 * *$ & $68,8 * *$ & 34,7 \\
\hline $\mathrm{P} 2+$ & $62,2 * *$ & 36,4 & 48,1 & $10,8 * *$ & 35,7 & $5,9 * *$ & $90,2 * *$ & 30,2 & $25,0 * *$ & 40,4 \\
\hline P2- & $4,4^{*}$ & 4,5 & 7,4 & $83,8 * *$ & 21,4 & 11,8 & $4,9 * *$ & 9,5 & $2, I * *$ & 15,9 \\
\hline P2-- & $\mathrm{II}, \mathrm{I}$ & 9,1 & 0,0 & 2,7 & 14,3 & $82,4 * *$ & $0,0 * *$ & 3,2 & 4,2 & 8,9 \\
\hline \multicolumn{11}{|l|}{ 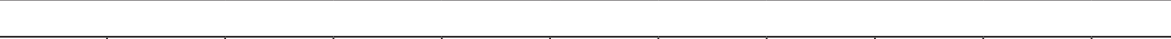 } \\
\hline $\mathrm{P} 3++$ & $0,0^{* *}$ & 4,5 & $96,3 * *$ & 8,6 & \begin{tabular}{|l|}
30,8 \\
\end{tabular} & 6,3 & $0,0 * *$ & $0,0 * *$ & $2,1 *$ & 11,8 \\
\hline P3+ & $67,4 * *$ & $9, I^{* *}$ & $0,0 * *$ & 45,7 & 23,1 & 31,3 & 51,3 & 38,7 & 29,2 & 37,0 \\
\hline P3- & 20,9 & $54,5 * *$ & $3,7 * *$ & 25,7 & 30,8 & 12,5 & 33,3 & $56,5 * *$ & $10,4^{* *}$ & 29,5 \\
\hline P3-- & 11,6 & 31,8 & $0,0 * *$ & 20,0 & 15,4 & $50,0 * *$ & 15,4 & $4,8^{* *}$ & $58,3 * *$ & 21,6 \\
\hline \multicolumn{11}{|l|}{ 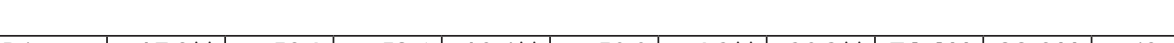 } \\
\hline P4++ & $17,8^{* *}$ & 59,1 & 53,6 & $19,4 * *$ & 50,0 & $6,2^{* *}$ & $29,3 * *$ & $76,6 * *$ & $88,0 * *$ & 49,4 \\
\hline P4+ & $75,6 * *$ & 40,9 & 46,4 & 22,2 & $7,1 * *$ & 12,5 & $68,3 * *$ & $23,4 * *$ & $10,0^{* * *}$ & 36,4 \\
\hline P4- & 6,7 & 0,0 & 0,0 & $50,0 * *$ & $42,9 * * *$ & 12,5 & 2,4 & $0,0 * *$ & $0,0 * *$ & 9,5 \\
\hline P4-- & 0,0 & 0,0 & 0,0 & 8,3 & 0,0 & 68,8 & 0,0 & 0,0 & 2,0 & 4,7 \\
\hline $\mathrm{P} 5++$ & 171 & 00 & & $0 *$ & $7 * *$ & & & & & 101 \\
\hline P5+ & $61,0 * *$ & $90.9 * *$ & $\begin{array}{r}4,7 \\
40,7\end{array}$ & $85,7 * *$ & $\frac{05,1}{7,2 * *}$ & $\frac{0}{31,3}$ & $\begin{array}{r}2,4 \\
36,6\end{array}$ & $\frac{1,0}{16 / * *}$ & $\frac{0,0 * \pi}{80^{* * *}}$ & $\frac{10,1}{393}$ \\
\hline P5- & $19,5^{*}$ & 4,6 ** & 44,5 & $11,4 * *$ & 0,0 ** & 12,5 & $56,1 * *$ & $74,2 * *$ & $20,0 * *$ & 34,4 \\
\hline P5-- & $2,4 * *$ & 4,5 ** & 7,4 & $2,9^{*}$ & 7,1 & 6,3 & $4,9 *$ & 8,1 & $72,0 * *$ & 16,2 \\
\hline \multicolumn{11}{|c|}{ Propositions relatives à la concurrence entre les établissements } \\
\hline $\mathrm{P} 6++$ & 0,0 & 0,0 & 0,0 & 2,9 & $38,4 * *$ & $23,5 * *$ & 0,0 & 0,0 & 2,1 & 3,5 \\
\hline $\mathrm{P6+}+$ & $33,3 *$ & $40,9 *$ & 11,5 & $5 I, 4 * *$ & 30,8 & $52,9 * *$ & $0,0 * *$ & $4,6 * *$ & $2,0 * *$ & 19,8 \\
\hline P6- & $60,0 * *$ & 10,9 & 23,1 & 28,6 & 15,4 & 11,8 & 29,3 & $52,3 * *$ & $2,0 * *$ & 32,9 \\
\hline P6-- & $6,7 * *$ & $18,2 * *$ & 65,4 & $17,1 * *$ & $15,4 * *$ & $11,8 * *$ & $70,7 * *$ & 43,1 & $93,9 * *$ & 43,8 \\
\hline \\
\hline P7++ & $6,8 * *$ & 23,8 & 55,6 & $11,8^{* *}$ & 28,6 & $6,7^{* *}$ & 53,7 & 46,9 & $87,5 * *$ & 40,9 \\
\hline P7+ & $88,6 * *$ & 66,7 & 44,4 & $44, I$ & $14,3^{*}$ & $13,3^{* *}$ & 41,5 & 50,0 & $12,5^{* *}$ & 45,1 \\
\hline P7- & 4,5 & 9,5 & 0,0 & $44, I * *$ & $57,1 * *$ & 20,0 & 4,8 & $1,5 * *$ & $0,0 * *$ & 10,7 \\
\hline \multicolumn{11}{|c|}{$60,0 * *$} \\
\hline $\mathrm{P} 8++$ & 00 *** & $773 * *$ & 74 & 143 & 231 & 235 & 25 & 00 *** & ก $0 * *$ & 104 \\
\hline P8+ & $86,4 * *$ & $4,5 * *$ & $51,9 *$ & $62,9 * *$ & 38,5 & 29,4 & $5,0 * *$ & $12,5 * *$ & 8,5 ** & 32,0 \\
\hline P8- & $11,4 * *$ & 9,1 & & $\mid I, 4^{*}$ & 15,4 & 11,8 & 42,5 & $76,6 * *$ & $2, I * *$ & 28,2 \\
\hline P8-- & $2,2 * *$ & 9,1 & 22,2 & $11,4^{* *}$ & 23,1 & 35,3 & $50,0 * *$ & $10,9 * *$ & $89,4 * *$ & 29,4 \\
\hline & & & & & & & & & & \\
\hline $\mathrm{P9++}$ & $18,2^{* *}$ & $90,0 * *$ & 44,5 & 52,9 & $71,4 * *$ & 50,0 & $9,8 * *$ & 27,0 & 40,4 & 37,3 \\
\hline $\mathrm{Pg}+$ & $81,8 * *$ & $10,0 * *$ & 37,0 & 41,2 & 21,4 & $12,5^{* *}$ & 34,1 & $68,3 * *$ & $25,5 * *$ & 44,4 \\
\hline P9- & $0,0 * *$ & 0,0 & 14,8 & 5,9 & 7,2 & 0,0 & $51,2 * *$ & $1,6 * *$ & 4,3 & 10,1 \\
\hline P9-- & $0,0 *$ & 0,0 & 3,7 & 0,0 & 0,0 & $37,5 * *$ & 4,9 & 3,1 & $29,8 * *$ & 8,2 \\
\hline
\end{tabular}

Lecture : $58,8 \%$ des personnels de direction de la classe 6 sont tout à fait d'accord avec la proposition PI contre $6,9 \%$ pour l'ensemble de l'échantillon (PI : Ce processus de "désectorisation" est une bonne chose parce que cela permet aux familles de choisir plus librement l'établissement dans lequel elles souhaitent scolariser leurs enfants.). Les résultats en caractères gras témoignent d'une surreprésentation. Ceux qui sont en italiques révèlent une sous-représentation.

** Khi-deux significatif à un seuil $\mathrm{P}<1 \%$

* Khi-deux significatif à un seuil $\mathrm{P}<5 \%$ 